\title{
Low-energy unphysical saddle in polynomial molecular potentials
}

\author{
Alessio Del Monte ${ }^{1}$, Nicola Manini ${ }^{1,2}$, \\ Luca Molinari ${ }^{1,3 *}$ and Gian Paolo Brivio ${ }^{4}$ \\ ${ }^{1}$ Dipartimento di Fisica, Università degli Studi di Milano, \\ Via Celoria 16, I-20133 Milano, Italy \\ ${ }^{2}$ INFM, Unità di Milano, Via Celoria 16, Milano, Italy \\ ${ }^{3}$ INFN, Sezione di Milano, Via Celoria 16, Milano, Italy \\ ${ }^{4}$ Dipartimento di Scienza dei Materiali, \\ Università di Milano-Bicocca, Via Cozzi 53, I-20125 Milano, \\ and INFM, Unità di Milano-Bicocca, Milano, Italy
}

Oct.29, 2004

\begin{abstract}
Vibrational spectra of polyatomic molecules are often obtained from a polynomial expansion of the adiabatic potential around a minimum. For several molecules, we show that such an approximation displays an unphysical saddle point of comparatively small energy, leading to a region where the potential is negative and unbounded. This poses an upper limit for a reliable evaluation of vibrational levels. We argue that the presence of such saddle points is general.
\end{abstract}

\section{Introduction}

The morphology of the adiabatic potential energy surface (APES), especially its low-energy minima and saddle points, is at the basis of the quantum chemistry of reaction paths and conformational transitions [1]. The adiabatic potential governs the low-energy vibrational dynamics of a rigid molecule of

*Author for correspondence. e-mail: luca.molinari@mi.infn.it 
$N$ atoms and is a complicate function of $d=3 N-6$ internal coordinates, such as bond lengths, bending or torsion angles $(d=3 N-5$ for linear molecules). Its actual determination is usually a very demanding problem [2], as the information contents of a function of $d$ coordinates grows exponentially with d. Global parametrizations of the APES computed with ab-initio methods are presently accessible for small molecules [3, 4, 5, 6]. A standard local parametrization, which was the mainroad to vibrational dynamics and is now mostly employed for medium-sized molecules[7], is provided by a Taylor expansion around the global minimum of the APES. For a deep minimum, one can isolate the vibrational contribution to the Hamiltonian, and expand it in normal modes 8 :

$$
\begin{aligned}
H= & T+V=\frac{1}{2} \sum_{a} \hbar \omega_{a}\left(p_{a}^{2}+q_{a}^{2}\right) \\
& +\frac{1}{3 !} \sum_{a b c} \phi_{a b c} q_{a} q_{b} q_{c}+\frac{1}{4 !} \sum_{a b c d} \phi_{a b c d} q_{a} q_{b} q_{c} q_{d}+\ldots
\end{aligned}
$$

$q_{a}$ are the dimensionless normal-mode coordinates measured with respect to the equilibrium geometry, and $p_{a}$ are the conjugate momenta. The frequencies $\omega_{a}$ and higher-order constants $\phi_{a b \ldots}$ of several molecules have been computed ab-initio, and sometimes refined by comparison with spectroscopic data [9]. For a simple molecule such as water, the anharmonic constants have been determined up to sixth order [10], and for several polyatomic molecules the literature reports calculations of third and fourth-order constants [11, 12, 13, 14, 15, 16, 17]. It is widely recognized that the Taylor series should be used with caution, since it only converges within a limited radius [18. The truncated series provides a locally accurate parametrization of the actual APES. Hereafter, we shall refer to the (finite) polynomial potential $V(q)$ in equation (1) as to the PP.

We consider the available data for the PP of several molecules. In all cases we find a saddle point of comparatively small energy, leading to an unphysical dissociative region where the potential is not bounded from below. We also find that, for most molecules, the potential well accomodates very few quantum levels up to the saddle energy. To our surprise, this problem seems to be neglected: its analysis is the main purpose of the present study.

A main use of the polynomial expansion (11) is the calculation of a number of vibrational levels, usually by means of perturbation or variational theories, or by numerical diagonalization. Contrary to finite-order perturbative calculations, a non-perturbative determination of vibrational levels inevitably detects tunneling to the unphysical region through the saddle. We show that sharp vibrational levels do occur only in the energy region below a quantum 
energy border, given by the sum of the energy of the lowest saddle (the classical border) plus the zero-point energy of the $d-1$ "transverse" modes (of positive curvature) at the saddle point.

The paper is organized as follows: In Sec. 2 we present the data for the lowest unphysical saddle point of several molecules, based on ab-initio anharmonic constants available in the literature, and give arguments for the general occurrence of a low-energy saddle in polynomial approximations of the molecular APES. In Sec. 3 we introduce the quantum energy border for the tunneling regime, and illustrate its influence on the vibrational spectra of two molecules which respond very differently, water and ketene. We employ a non-perturbative method developed by us for evaluating the spectra, which is presented in the Appendix. In Sec. 4 we discuss the results and the ensuing scenario.

\section{The unphysical saddle}

A unique feature of the polynomial expansion in equation (11) for the special case of diatomic molecules $(d=1)$ is that many even-power terms are positive (for example all even-power terms are positive for the Morse and Lennard-Jones functions). As a consequence, the truncation of the series to a positive even-power coefficient gives a lower-bounded potential, and thus a well-defined quantum problem, characterized by an infinite set of discrete levels. This feature of $d=1$ is unfortunately lost when the power expansion (1) is extended to polyatomic molecules $(d>1)$ : in all systems which we could obtain the anharmonic parameters for, we verified the occurrence of regions where the fourth-order $\mathrm{PP}$ is unbounded below.

\section{[Insert figure 1 about here]}

Energy barriers separate different minima of a physical APES, corresponding to different local equilibrium configurations (isomers) of the molecule. The isomerization dynamics occurs mainly via quantum tunneling or thermal activation through the lowest saddle of the barrier [19, 20]. Likewise, for the PP, energy barriers separate the region of the physical minimum around which the expansion is based and well grounded, from the unphysical regions where the potential drops to $-\infty$. The escape to the unphysical region is driven by the lowest saddle, which introduces an energy "border" that limits the range for (meta-stable) quantum levels allowed in the physical well of the PP. Figure 1 illustrates this concept in a simple $d=1$-dimensional context, where we purposedly truncated the polynomial expansion (1) to an odd order. It is clear that the saddle lies in a region where the polynomial has already become a poor approximation to the actual APES. 
To determine the lowest saddle point of the PP of a polyatomic molecule, we first locate all stationary points in the neighborhood of $\mathbf{q}=\mathbf{0}$. For this purpose, we compute analytically the gradient $\nabla_{\mathbf{q}} V$, and repeatedly solve the polynomial equation $\nabla_{\mathbf{q}} V=\mathbf{0}$ by the Newton method, starting from a scattered set of several thousand random initial points. This procedure generates a large number of stationary points of the PP. We then select the solution $\mathbf{q}_{\mathrm{s}}$ with the lowest positive energy $E_{\mathrm{s}}$ and check that it is indeed a saddle point, characterized by one negative and $d-1$ positive curvatures. Finally we verify that the $\mathrm{PP}$, restricted to the straight line through the points $\mathbf{q}=\mathbf{0}$ and $\mathbf{q}_{\mathrm{s}}$, has a shape qualitatively similar to the dashed line of figure 1, i.e. that tunneling indeed occurs through a single barrier to a region where the potential drops to $-\infty$.

\section{[Insert table 1 about here]}

For several molecules, table 1 reports the height $E_{\mathrm{s}}$ of the lowest (unphysical) saddle point of the PP, measured from the bottom of the potential well. Surprisingly, these saddles are low: only about few times a typical harmonic vibrational frequency of the molecule. As a result, few, if any, vibrational states sit below $E_{\mathrm{s}}$. The reported values of $E_{\mathrm{s}}$ of the diatomic molecules 21] set the characteristic scale given by the bond anharmonicities, in the $10^{4} \mathrm{~cm}^{-1}$ region. This can be regarded as an upper bound, as off-diagonal anharmonicities of the PP can only provide lower saddles, involving several normal coordinates at the same time. Indeed table 1 shows lower saddles for polyatomic molecules of increasing number of atoms. Especially low saddles are found for molecules characterized by soft torsional modes, such as methanol $\mathrm{CH}_{3} \mathrm{OH}$. The difference in energy between the two saddle heights obtained from different available $\mathrm{PP}$ of $\mathrm{H}_{2} \mathrm{O}$ suggests an estimate of the accuracy of the reported values of $E_{\mathrm{s}}$ due to the approximations involved in $a b$-initio calculations of the anharmonic parameters.

The occurrence of a saddle leading to an unphysical region is by no means specific of the PP of the molecules listed in table 1: we argue that this is a general feature to be found in the PP of most polyatomic molecules. Indeed, also for an even-power truncation, the PP can easily drop to $-\infty$ in some direction in q space 1 . The precise value of the 4 th-order parameters $\phi_{a b c d}$ (including their sign) is determined by the local properties of the physical APES at its minimum $\mathbf{q}=\mathbf{0}$, not by any requirement of confining behaviour at large distance: the PP of a real molecule easily contains negative semi-diagonal

\footnotetext{
1 For example, the fourth-order terms $\phi_{1111} q_{1}^{4}+\phi_{1222} q_{1} q_{2}^{3}+\phi_{2222} q_{2}^{4}$ combine to $\left(\gamma^{4} \phi_{1111}+\gamma \phi_{1222}+\phi_{2222}\right) q_{2}^{4}$ along the line $q_{1}=\gamma q_{2}$, and the numeric coefficient $\gamma^{4} \phi_{1111}+\gamma \phi_{1222}+\phi_{2222}$ can easily be negative, provided that $\left|\phi_{1222}\right|$ is large enough and that $\gamma$ is chosen suitably. Also, to make things worse, even though fully diagonal $\phi_{a a a a}$ terms are usually positive, there often occur semi-diagonal terms $\phi_{a a b b}$ with negative sign.
} 
terms $\phi_{a a b b}$ and sizable mixed terms $\phi_{a b c d}$, which in turn produce regions where the PP drops to $-\infty$. In practice, the same argument prevents confining behaviour also of 6 th and higher even-order terms, and an even-power truncated PP does not behave any better, away from the physical minimum, than an odd-power truncated PP. Therefore, we consider it extremely unlikely (though technically possible) that a real polyatomic molecular potential may ever be found whose polynomial expansion at the minimum (truncated at any order $>2$ ) is lower-bounded everywhere. Several methods to circumvent the incorrect behavior of polynomial approximations at large $\mathbf{q}$ are commonly employed: for example, Morse coordinates for stretching modes are used to correct the unphysical power-law asymptotics of the PP [22, 23, 24].

\section{The quantum energy border}

As the PP has no lower bound, the associated Schrödinger problem is illdefined. However, resonant states with complex energy values $E_{a}-i \Gamma_{a}$ usually exist in the well [25, 19, 26, 27, 28, 29, 30, 31]. Deep in the well, $\Gamma_{a}$ is in general exponentially small and proportional to the Gamow factor $e^{-2 S / \hbar}$, where $S$ is the imaginary-time action along the most probable tunneling path inside the barrier. Resonances appear as sharp peaks in the spectral density, at energies $E_{a}$ extremely close to the eigenenergies of the Schrödinger equation restricted to the well. As energy increases toward the threshold value, tunneling evolves into a "leaking" regime, characterized by the appearence of resonances whose imaginary part $\Gamma_{a}$ is comparable to the real part [30], relics of further excited states in the well, strongly hybridized to the continuum.

In one dimension, the threshold coincides with the saddle energy $E_{\mathrm{s}}$. For $d>1$, an effect absent in the simple one-dimensional picture of figure 1 is to be considered: tunneling through the barrier at the saddle point is hindered by the "transverse" motion of the degrees of freedom perpendicular to the one crossing the barrier. These perpendicular degrees of freedom are associated to a minimum energy $E_{\mathrm{zp}}^{\perp}$ due to Heisenberg's uncertainty, that adds to the saddle height to determine the quantum energy border between the tunneling and the leaking regimes

$$
E_{\mathrm{qb}}=E_{\mathrm{s}}+E_{\mathrm{zp}}^{\perp} .
$$

As the study of tunneling problems [32, 33] suggests, we approximate $E_{\mathrm{zp}}^{\perp}$ by its harmonic expression

$$
E_{\mathrm{zp}}^{\perp} \simeq \frac{1}{2} \sum_{i=2}^{d} \hbar \omega_{i}^{\prime}
$$

in terms of the $d-1$ real harmonic frequencies $\omega_{i}^{\prime}$ at the saddle point $\left(\omega_{1}^{\prime}\right.$ 
is the imaginary frequency associated to the tunneling direction). Since, for most polyatomic molecules, $E_{\mathrm{zp}}^{\perp}$ is close to the zero-point energy of the ground state $E_{\mathrm{zp}}(\mathbf{0})$ (see table 1 ), the raising of the energy border due to $E_{\mathrm{zp}}^{\perp}$ recovers a spectral range $\left(E_{\mathrm{zp}}(\mathbf{0})<E<E_{\mathrm{qb}}\right)$ where quasistationary vibrational levels are to be found, of extension comparable to the classical region for bounded motion $\left(0 \leq E \leq E_{\mathrm{s}}\right)$.

To illustrate the difficulties brought in by the unphysical saddle in polynomial approximations of APES, we compute non-perturbatively the vibrational spectra of two molecules, water and ketene, with different outcomes. For both, we employ ab-initio quartic anharmonic potentials for the normal modes [10, 34]: while the PP of the water molecule features an energy range between the zero point energy and the quantum border containing a number of vibrational levels, for ketene the range is so small to contain very few levels. We evaluate the levels $\left|E_{a}\right\rangle$ of the vibrational Hamiltonian $H$ (1) by means of a non-perturbative technique equivalent to an exact diagonalization on a finite basis. Its advantage over standard Lanczos/Davidson diagonalization is a uniform precision throughout the whole spectrum. The Green function

$$
G(E+i \varepsilon)=\left\langle\mathbf{v}_{0}\left|(E+i \varepsilon-H)^{-1}\right| \mathbf{v}_{0}\right\rangle
$$

is computed with an iterative procedure on a set of values $E$. The initial state $\left|\mathbf{v}_{0}\right\rangle$ is chosen as an eigenstate of the harmonic part of $H$. The spectrum is obtained from the lineshape function

$$
I(E)=-\frac{1}{\pi} \operatorname{Im} G(E+i \varepsilon)=\frac{\varepsilon}{\pi} \sum_{a} \frac{\left|\left\langle E_{a} \mid \mathbf{v}_{0}\right\rangle\right|^{2}}{\left(E-E_{a}\right)^{2}+\varepsilon^{2}} .
$$

Eigenvalues show up as peaks of $I(E)$, with heights proportional to the squared overlap of the exact eigenstates to the initial excitation. The parameter $\varepsilon$ introduces a phenomenological Lorentzian broadening of the lines $E_{a}$.

A few words on the method for evaluating the Green function are necessary. First, one constructs a basis of harmonic states grouped into families $T_{i}$ (tiers), $i=0 \ldots N$, adapted to the specific PP and the initial state $\left|\mathbf{v}_{0}\right\rangle$ [35, 36, 37]. Next, the Green function is evaluated through the exact recursive relation equation (8), as detailed in the Appendix. As the basis is finite, the spectrum consists of a finite number of real eigenvalues. By evaluating the spectra $I(E)$ with incresing number of tiers $N$, one has a control of convergence in the regions below the quantum border, where tunneling is suppressed. Above the border, no convergence is expected as the basis size is changed. 


\subsection{Water}

We employ the ab-initio quartic PP parameters listed in table 6 of Ref. [10]. The two real frequencies $\hbar \omega_{2}^{\prime}=4758 \mathrm{~cm}^{-1}$ and $\hbar \omega_{3}^{\prime}=4849 \mathrm{~cm}^{-1}$ at the lowest saddle point of $\mathrm{H}_{2} \mathrm{O}\left(E_{\mathrm{s}}=6846 \mathrm{~cm}^{-1}\right)$ are found so much larger than the three curvatures at the minimum, that $E_{\mathrm{zp}}^{\perp}>E_{\mathrm{zp}}(\mathbf{0})$ (table 1): this produces a rather narrow saddle, which pushes the quantum border up to $E_{\mathrm{qb}}=11649 \mathrm{~cm}^{-1}$.

\section{[Insert figure 2 about here]}

We take the harmonic fundamental excitation of the $\omega_{1}$ (symmetric $\mathrm{OH}$ stretching) mode as initial state $\left|\mathbf{v}_{0}\right\rangle$, and obtain the spectrum in figure 2 . The peaks, representing exact vibrational levels of the PP, are assigned to the harmonic quantum numbers of the closest state resulting from standard second-order perturbation theory. Convergence is studied by increasing the tier number from $N=3$ to $N=15$. It is very good already using $N=3$ tiers, thus showing the effectiveness of the tiers Green-function method. Hardly any tunneling is observed below $E_{\mathrm{qb}}$. Across $E_{\mathrm{qb}}$, the appearence of the weak non-converging satellites confirms that the resonances in this region are affected by detectable leakage to the continuum. Indeed, since the lowest saddle lies in a direction involving mainly mode 1 , the chosen initial state $|1,0,0\rangle$ promotes tunneling. Nonetheless, the stabililty of several features even above $E_{\mathrm{qb}}$ indicates that a number of fairly long-lived physical states are so localized in the well that their overlap to the outside continuum is relatively small.

\section{$3.2 \quad$ Ketene}

As shown in table 1, according to the ab-initio calculation of Ref. 34 the lowest saddle of the PP of ketene $\mathrm{H}_{2} \mathrm{C}_{2} \mathrm{O}$ is very low. The resulting useful energy range is therefore extremely narrow, from $E_{\text {zp }}(\mathbf{0}) \simeq 6900 \mathrm{~cm}^{-1}$ to $E_{\mathrm{qb}} \simeq 7500 \mathrm{~cm}^{-1}$. Only the ground state and the fundamental excitations of the lowest bending modes are located in the spectral region below the quantum border. The PP of ketene represents therefore a particulary unfavorable case where no convergence is expected for any excitation.

\section{[Insert figure 3 about here]}

Figure 3 shows the spectral region from the ground state to the $\mathrm{CH}$ stretching mode, obtained with the $a_{1}$ initial excitation $|1,0,0,0,0,0,0,0,0\rangle$ of the $\mathrm{CH}$ symmetric stretching mode $\nu_{1}$, for different sizes of the basis. In this region, only a few fundamental and overtone/combination states of $a_{1}$ symmetry should be found. Instead, tens of spurious structures arise, which show no tendency to converge as the basis size increases. This is to be con- 
trasted with the converging spectral range of $\mathrm{H}_{2} \mathrm{O}$, where a stable spectrum is achieved already with a small basis of $N=3$ tiers.

\section{Discussion}

We show that polynomial approximations of molecular potentials usually display unphysical saddles that lead to regions where the potential is not lower-bounded. The height of the saddle and the zero-point energy of transverse modes both determine a quantum energy border. Unless the saddle is very low, as e.g. in the $\mathrm{PP}$ of the ketene molecule, in the spectral region below the quantum border tunneling is exponentially small and standard perturbative treatment of the anharmonic interactions usually provides a good approximation to the sharp level positions. Perturbative calculations are often extended to higher energies, based on the general belief that, like in the $d=1$ Morse case, the second-order approximation compensates the wrong behaviour of the PP away from the minimum and reproduces the levels of a physical APES [38].

Our non-perturbative calculations show that, as energy is raised above the quantum border, resonances leave the tunneling regime and couple more and more strongly to the continuum, practically washing out all spectral details. Such highly excited states are of scarce physical relevance anyway, since the extension of the associated wavefunction explores regions where the PP becomes a very poor approximation of the true APES.

The present results cast a shadow on the traditional use of the polynomial approximation of the physical APES for the calculation of highly excited vibrational or roto-vibrational spectra of polyatomic molecules. For example, in most molecules, IVR (intramolecular vibrational relaxation) spectra involve energy levels much above the saddle [39]. Hence, IVR applications of exact numerical methods, such as the Lanczos or Davidson algorithms [40, 41, 42, 43] or the Green-function method proposed here, are bound to face the problem of the saddle of the PP. The consequent broadening of the resonant states poses an intrinsic limit to the spectral accuracy which can be obtained. A real progress may be achieved by the use of smarter local parametrizations of the APES (e.g. based on Morse coordinates [23, 24]).

\section{Acknowledgements}

We thank J.H. van der Waals, G. Scoles, K. Lehmann, A. Callegari for useful discussions, and J.M.L. Martin, J.P. François for kindly providing us the PP 
parameters for ethylene.

\section{A Non-perturbative evaluation of the Green function.}

We present a general procedure, inspired to Ref. [44] and there indicated as "tiers method", for the non-perturbative evaluation of the eigenvalues and spectral weights of a Hamiltonian decomposed as $H=H_{0}+H^{\prime}$. For definiteness, we consider the problem at hand: $H_{0}=\sum \hbar \omega_{b}\left(a_{b}^{\dagger} a_{b}+1 / 2\right)$ describes $d$ independent oscillators, with eigenvectors $|\mathbf{v}\rangle=\left|v_{1}, v_{2}, \ldots, v_{d}\right\rangle$, $H^{\prime}$ is the anharmonic part of the potential (1).

The first step of the method is to partition the unperturbed eigenvectors in families (tiers) $T_{0}, T_{1}, \ldots$ of decreasing perturbative relevance, such that the matrix representation of $H$ is block-tridiagonal in the tiers. Symbolically we write the blocks as $H_{i i}=\left\langle T_{i}|H| T_{i}\right\rangle$ and $H_{i, i+1}=\left\langle T_{i}|H| T_{i+1}\right\rangle$. Depending on the problem under investigation, a set of unperturbed states $\left|\mathbf{v}_{0, \alpha}\right\rangle$ ( $\alpha=$ $\left.1, \ldots, t_{0}\right)$ is selected to form the initial tier $T_{0}$. In the computations of this paper, $T_{0}$ contains a single initial state. The action of $H^{\prime}$ on $T_{0}$ gives new vectors: the basis states that have non-zero overlap with them, and are not in $T_{0}$, are collected in $T_{1}$. We label them as $\left|\mathbf{v}_{1, \alpha}\right\rangle\left(\alpha=1 \ldots t_{1}\right)$. For a finite set $T_{0}$, and an interaction $H^{\prime}$ which is a polynomial in the raising and lowering operators, tier $T_{1}$ and subsequent ones are finite. Next we consider the set $H^{\prime} T_{1}$, and expand it in the eigenvectors already in $T_{0}$ and $T_{1}$, plus new ones that are collected in tier $T_{2}$. The process is iterated to produce further tiers $T_{3}, T_{4}, \ldots$ Up to this point the method is simply a smart algorithm to generate systematically a good approximate basis for a quantum problem where some non-interacting part $H_{0}$ of the Hamiltonian can be singled out. Indeed, similar basis generation has been employed successfully in different contexts [44, 41, 43, 45, 46]. However, the hierarchical basis structure and the corresponding block-tridiagonal form of the Hamiltonian, suggest a natural iterative method to construct the spectrum.

The matrix element of the resolvent $\mathcal{G}(z)=(z I-H)^{-1}$ in $T_{0}$ is precisely the Green function in equation (4). We propose to compute it based on the following formula for the inversion of partitioned matrices, with square diagonal blocks (we omit unneeded terms):

$$
M=\left(\begin{array}{ll}
M_{11} & M_{12} \\
M_{21} & M_{22}
\end{array}\right), \quad M^{-1}=\left(\begin{array}{cc}
{\left[M_{11}-M_{12}\left(M_{22}\right)^{-1} M_{21}\right]^{-1}} & \ldots \\
\ldots & \ldots
\end{array}\right)
$$

We apply this formula by identifying $M$ with the matrix representations of $z I-H$ and $M^{-1}$ with the resolvent $\mathcal{G}(z)$. The 4 blocks result from the 
separation of the basis into the set $T_{0}$ and the ordered set $T_{1} \cup T_{2} \cup \ldots$ Offdiagonal matrix elements of $M$ are due to $H^{\prime}$ only. Thus $M_{11}=z I_{0}-H_{00}$ ( $I_{0}$ is the unit matrix of size $t_{0}$ and $H_{00}=\left\langle T_{0}|H| T_{0}\right\rangle$ ). $M_{22}$ is the matrix $(z I-H)$ expanded in the remaining tiers. $M_{12}=M_{21}^{t}$ is a rectangular matrix of size $t_{0} \times\left(t_{1}+t_{2}+\ldots\right)$. By the tier construction, non-zero matrix elements of $M_{12}$ are restricted to the leftmost submatrix of size $t_{0} \times t_{1}$, that identifies with $-H_{01}^{\prime}=-\left\langle T_{0}\left|H^{\prime}\right| T_{1}\right\rangle$.

The aim of the calculation is to evaluate the block $\left(M^{-1}\right)_{11} \equiv G(z) \equiv$ $G^{(0)}(z)=\left\langle\mathbf{v}_{0}|\mathcal{G}(z)| \mathbf{v}_{0}\right\rangle$. The inversion formula (6) provides

$$
G^{(0)}(z)=\left[z I_{0}-H_{00}-H_{01}^{\prime} G^{(1)}(z) H_{10}^{\prime}\right]^{-1},
$$

where the $t_{1} \times t_{1}$ matrix $G^{(1)}(z)=\left\langle T_{1}\left|\left(M_{22}\right)^{-1}\right| T_{1}\right\rangle$. To evaluate it we use equation (6) again, with the blocks now resulting from the separation of the basis into the set $T_{1}$ and the set $T_{2} \cup T_{3} \cup \ldots$ Now $M_{11}=\left(z I_{1}-H_{11}\right)$ and $M_{22}$ is the matrix $(z I-H)$ expanded in the basis $T_{2} \cup T_{3} \cup \ldots$ The matrix $G^{(1)}(z)$ coincides with the block $\left(M^{-1}\right)_{11}$ :

$$
G^{(1)}(z)=\left[z I_{1}-H_{11}-H_{12}^{\prime} G^{(2)}(z) H_{21}^{\prime}\right]^{-1}
$$

where $G^{(2)}(z)=\left\langle T_{2}\left|\left(M_{22}\right)^{-1}\right| T_{2}\right\rangle$. By iterating the same inversion formula (6) we obtain a chain of relations of the type

$$
G^{(k-1)}(z)=\left[z I_{k-1}-H_{k-1, k-1}-H_{k-1, k}^{\prime} G^{(k)}(z) H_{k, k-1}^{\prime}\right]^{-1}
$$

In practice, the (in principle infinite) chain is truncated by approximately taking $G^{(N)}(z) \approx\left(z I_{N}-H_{N N}\right)^{-1}$. This is the only approximation involved in this method, and it amounts to neglecting the coupling of $T_{N}$ to the subsequent tier. Starting from $G^{(N)}(z)$, one iterates (8) back to the sought for matrix $G^{(0)}(z)$. This procedure is a matrix generalization of the continued fraction expansion for the inversion of tridiagonal matrices [47].

This method provides good evaluations of the position of the quasistationary states, including a rigorous treatment of all anharmonic resonances. These effects were mostly left out in the approximate treatment of Ref. [44], where off-diagonal terms $M_{12}$ in equation (66) were neglected. The recursive calculation of the Green function (8) has several advantages with respect to the more traditional Lanczos method [48, 49, 41, 45]: (i) it provides equal accuracy through the whole spectrum, while Lanczos method is more accurate close to the endpoints; (ii) it splits the Hilbert space into subspaces $T_{0}, \ldots T_{N}$ to treat one at a time; (iii) once the chain of matrices is set up, each frequency requires an independent calculation, which makes this method suitable for parallel calculations. Its main disadvantage is the rapid 
growth of the tier size $t_{i}$, for systems with many degrees of freedom. To fit the available $\mathrm{CPU} /$ memory limits, it is possible to cutoff the tier growth to some maximum size $t_{\max }$, as described in Ref. [45]. In general, the recursive method may become very costly in CPU time, since the evaluation of $G^{(0)}(E+i \varepsilon)$ requires $N$ inversions for each sample frequency $E$, each inversion costing a time proportional to $t_{\max }^{3}$. In the Lanczos method, a single chain of $N_{\text {Lanczos }} \approx 10^{3}$ iterations, each costing of the order of the Hilbert space size $\sim N \cdot t_{\max }$, generates the whole spectrum.

The $c^{++}$code for computing the tier basis and the spectrum based on the Green-function recursive inversion formula (8) is available in Ref. [50]. 


\section{References}

[1] Ramquet, M. N., Dive, G., and Dehareng, D., 2000, J. Chem. Phys. $\mathbf{1 1 2}, 4923$.

[2] Global, Accurate Vibration Hamiltonians from High-Resolution Molecular Spectroscopy; edited by M.Herman, J.Lievin, J. W. Auwera and A.Campargue, 1999, Adv. Chem. Phys., Vol. 108 (Wiley, New York 1999).

[3] Kuhn, B., Rizzo, T. R., Luckhaus, D., Quack, M., and Suhm, M. A., 1999, J. Chem. Phys. 111, 2565.

[4] Leforestier, C., Viel, A., Gatti, F., Muñoz, C., and Iung, C., 2001, J. Chem. Phys. 114, 2099.

[5] Carter, S. and Handy, N. C., 2002, Chem. Phys. Lett. 352, 1.

[6] Kurkal, V., Fleurat-Lessard, P., and Schinke, R., 2003, J. Chem. Phys. 119, 1489.

[7] Maslen, P. E., Handy, N. C., Amos, R. D., and Jayatilaka, D., 1992, J. Chem. Phys. 97, 4233.

[8] Bright Wilson, E., Decius, J. C., and Cross, P. C., 1955, Molecular Vibrations, The Theory of Infrared, Raman Vibrational Spectra (McGrawHill, New York).

[9] Ab initio derived force fields: state of the science, edited by T. J. Lee, 1997, Spectrochim. Acta A 53, Special Issue.

[10] Császár, A. G. and Mills, I. M., 1997, Spectrochim. Acta A 53, 1101.

[11] Č́ížek, J., Špirko, V., and Bludský, O., 1993, J. Chem. Phys. 99, 7331.

[12] Martin, J. M. L., Lee, T. J., Taylor, P. R., and François, J. P., 1995, J. Chem. Phys. 103, 2589.

[13] Koput, J. and Peterson, K. A., 1998, Chem. Phys. Lett. 283, 139.

[14] Miani, A., Cane, E., Palmieri, P., Trombetti, A., and Handy, N. C., 2000, J. Chem. Phys. 112, 248.

[15] Burcl, R., Handy, N. C., and Carter, S., 2003, Spectrochim. Acta A, 59, 1881 . 
[16] Demaison, J., Perrin, A., and Burger, H., 2003, J. Mol. Spectr. 221, 47.

[17] Yagi, K., Hirao, K., Taketsugu, T., Schmidt, M. W., and Gordon, M. S., 2004, J. Chem. Phys. 121, 1383.

[18] Steele, D., Lippincott, E. R., and Vanderslice, J. T., 1962, Rev. Mod. Phys. 34, 239251.

[19] Carrington, T., Hubbard, L. M., Schaefer III, H. F., and Miller, W. H., 1984, J. Chem. Phys. 4347.

[20] Hänggi, P., Talkner, P., and Borkovec, M., 1990, Rev. Mod. Phys. 62, 251.

[21] Bransden, B. H. and Joachain, C. J., 2003, Physics of Atoms and Molecules (Prentice Hall, Englewood Cliffs, NJ).

[22] Murrell, J. N. and Guo, H., 1987, J. Chem. Soc. Faraday II, 83, 683.

[23] Carter, S., Mills, I. M., and Handy, N. C., 1993, J. Chem. Phys. 99, 4379 .

[24] Zhou, C., Xie, D., Chen, R., Yan, G., Guo, H., Tyng, V., and Kellman, M. E., 2002, Spectrochim. Acta A 58, 727.

[25] Miller, W. H., Handy, C., and Adams, J. E., 1980, J. Chem. Phys. 72, 99.

[26] Hänggi, P., Weiss, U., and Riseborough, P., 1986, Phys. Rev. A 34, 4558 .

[27] Benderskii, V. A., Makarov, D. E., and Wight, C. A., 1994, Chemical Dynamics at low temperatures, Adv. Chem. Phys. Vol. 88 (Wiley, New York 1994).

[28] Zamastil, J., Špirko, V., Čížek, J., Skála, L., and Bludský, O., 2001, Phys. Rev. A 64, 042101.

[29] Miller, W. H., Ceotto, T. Zhao. M., and Yang, S., 2003, J. Chem. Phys. 119, 1329.

[30] Yaris, R., Bendler, J., Lovett, R. A., Bender, C. M., and Fedders, P. A., 1978, Phys. Rev. A 18, 1816.

[31] Rescigno, T. N., Baertschy, M., Byrum, D., and McCurdy, C. W., 1997, Phys. Rev. A 55, 4253. 
[32] Kitamura, H., Tsuneyuki, S., Ogitsu, T., and Miyake, T., 2000, Nature 404, 259.

[33] Miani, A., Hänninen, V., Horn, M., and Halonen, L., 2000, Mol. Phys. 98, 1737.

[34] East, A., Allen, W., and Klippenstein, S., 1995, J. Chem. Phys. 102, 8506 .

[35] Bixon, M. and Jortner, J., 1968, J. Chem. Phys. 48, 715.

[36] Uzer, T., 1991, Phys. Rep. 199, 73.

[37] Marshall, K. T. and Hutchinson, J. S., 1991, J. Chem. Phys. 95, 3232.

[38] Mills, I. M. and Robiette, A. G., 1985, Mol. Phys. 56, 743.

[39] Pearman, R. and Gruebele, M., 1998, J. Chem. Phys. 108, 6561.

[40] Gruebele, M., 1996, J. Chem. Phys. 104, 2453.

[41] Wyatt, R., 1998, J. Chem. Phys. 109, 10732.

[42] Pochert, J., Quack, M., Stohner, J., and Willeke, M., 2000, J. Chem. Phys. 113, 2719.

[43] Callegari, A., Pearman, R., Choi, S., Engels, P., Srivastava, H., Gruebele, M., Lehmann, K. K., and Scoles, G., 2003, Mol. Phys. 101, 551.

[44] Stuchebrukhov, A. A. and Marcus, R. A., 1993, J. Chem. Phys. 98, 6044 .

[45] Manini, N., Gattari, P., and Tosatti, E., 2003, Phys. Rev. Lett. 91, 196402.

[46] Wang, X. G. and Carrington, T., 2003, J. Chem. Phys. 119, 101.

[47] Haydock, R., 1980, The recursive solution of the Schrödinger equation, Solid State Physics, Vol 35 (Academic Press, New York).

[48] Jaklic, J. and Prelovsek, P., 2000, Adv. Phys. 49, 1.

[49] Köppel, H., Döscher, M., Bâldea, I., Meyer, H.-D., and Szalay, P. G., 2002, J. Chem. Phys. 117, 2657.

[50] Del Monte, A. and Manini, N., 2004, http://www.mi.infm.it/manini/ivr.html. 
Table 1: Lowest saddle-point energy $E_{\mathrm{s}}$, harmonic zero-point energy at the minimum $E_{\text {zp }}(0)$, harmonic transverse zero-point energy at the saddle point $E_{\mathrm{zp}}^{\perp}$, of the 4 th-order PP of several polyatomic molecules. For diatomics, the saddle energy $E_{\mathrm{s}}$ is replaced by the height of the maximum of the fifth-order Taylor expansion of a Morse potential fitting the experimental harmonic frequency and dissociation energy [21]. Energies are divided by $h c$ and expressed in traditional spectroscopic wavenumber units $\mathrm{cm}^{-1}$.

\begin{tabular}{lrrrr}
\hline \hline $\begin{array}{l}\text { molecular } \\
\text { species }\end{array}$ & $\begin{array}{r}E_{\mathrm{s}} \\
\mathrm{cm}^{-1}\end{array}$ & $\begin{array}{r}E_{\mathrm{zp}}(\mathbf{0}) \\
\mathrm{cm}^{-1}\end{array}$ & $\begin{array}{r}E_{\mathrm{zp}}^{\perp} \\
\mathrm{cm}^{-1}\end{array}$ & Ref. \\
\hline $\mathrm{N}_{2}$ & 32834 & 1180 & - & {$[21]$} \\
$\mathrm{HCl}$ & 14919 & 1495 & - & {$[21]$} \\
$\mathrm{H}_{2} \mathrm{O}$ & 6846 & 4717 & 4803 & {$[10]$} \\
$\mathrm{H}_{2} \mathrm{O}$ & 7208 & 4712 & 4488 & {$[11]$} \\
$\mathrm{H}_{2} \mathrm{~S}$ & 8529 & 3335 & 3488 & {$[11]$} \\
$\mathrm{NO}_{2}$ & 13698 & 1890 & 1936 & {$[11]$} \\
$\mathrm{SO}_{2}$ & 6263 & 1537 & 1270 & {$[11]$} \\
$\mathrm{HOF}_{\mathrm{HOCl}}$ & 4624 & 3038 & 3043 & {$[11]$} \\
$\mathrm{H}_{2} \mathrm{C}_{2} \mathrm{O}$ & 2821 & 2911 & 2893 & {$[13]$} \\
$\mathrm{C}_{2} \mathrm{H}$ & 834 & 6907 & 6641 & {$[34]$} \\
$\mathrm{CH}_{3} \mathrm{OH}$ & 3483 & 11151 & 10815 & {$[12]$} \\
\hline \hline
\end{tabular}


Figure 1: A typical 1-dimensional molecular potential (Morse, solid) and its 5th-order Taylor polynomial approximation (dashed) illustrating the presence of an unbounded region separated from the physical confining region by a barrier topping at a saddle point $\mathbf{q}_{\mathrm{s}}$.

Figure 2: The spectrum of $\mathrm{H}_{2} \mathrm{O}$ for initial excitation $|1,0,0\rangle$, computed with $N=3$ (294 states) and $N=15$ (16811 states), broadening $\varepsilon=4 \mathrm{~cm}^{-1}$. Below the quantum energy border (dashed line) convergence is satisfactory with relative error $\leq 1 \%$. The main features seem to converge also above $E_{\mathrm{qb}}$, but the appearance of new structures for larger $N$ indicates non-negligible leakage.

Figure 3: The spectrum of ketene for initial excitation $|1,0,0,0,0,0,0,0,0\rangle$, computed with $N=3$ (4299 states), $N=5$ (8299 states), and $N=10$ (18299 states), broadening $\varepsilon=4 \mathrm{~cm}^{-1}$. No convergence is observed, even below the quantum energy border, due to sizable leakage. The relatively stable feature near $10000 \mathrm{~cm}^{-1}$ corresponds to the energy position of the initial excitation. 


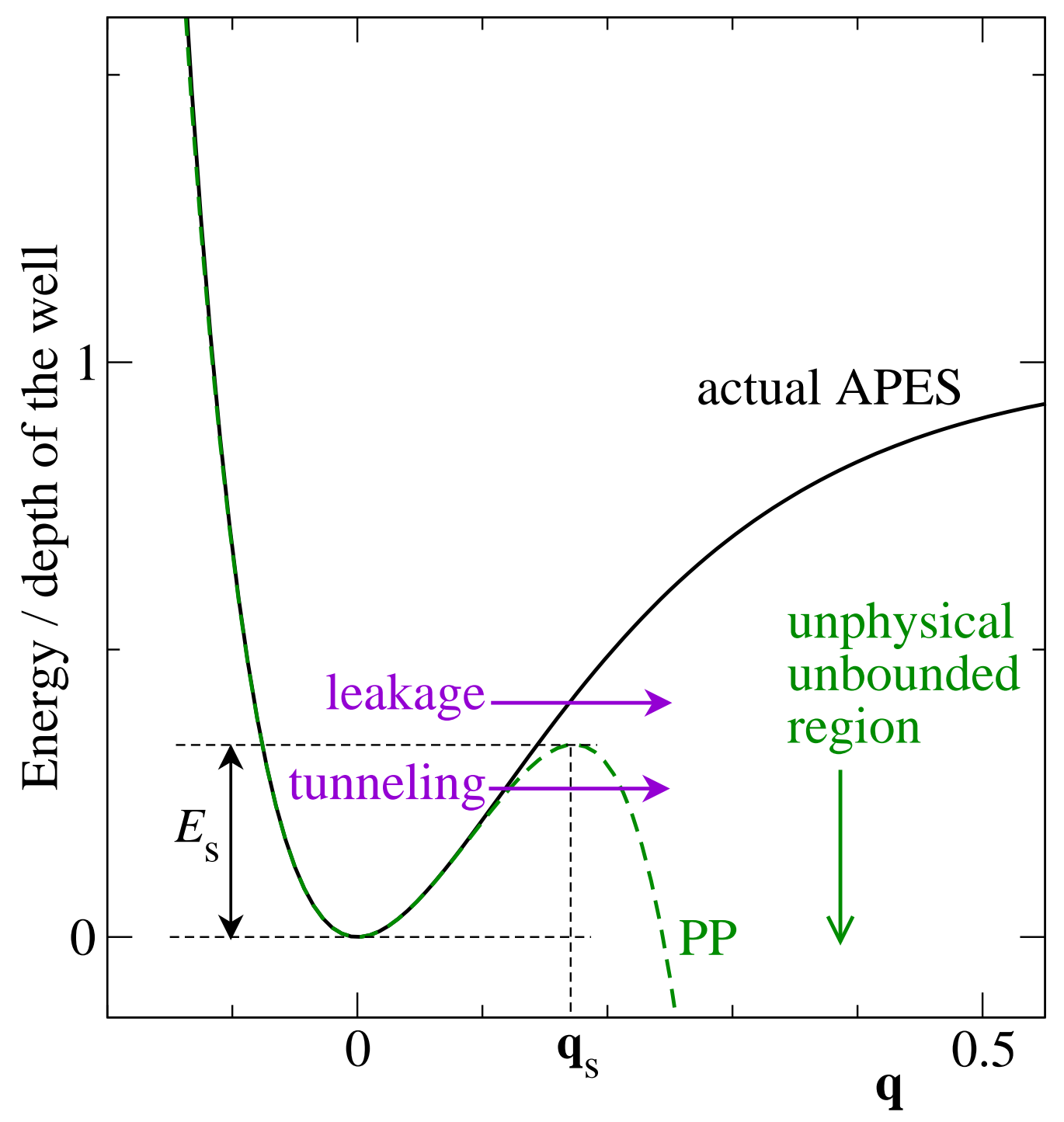

Figure 1 


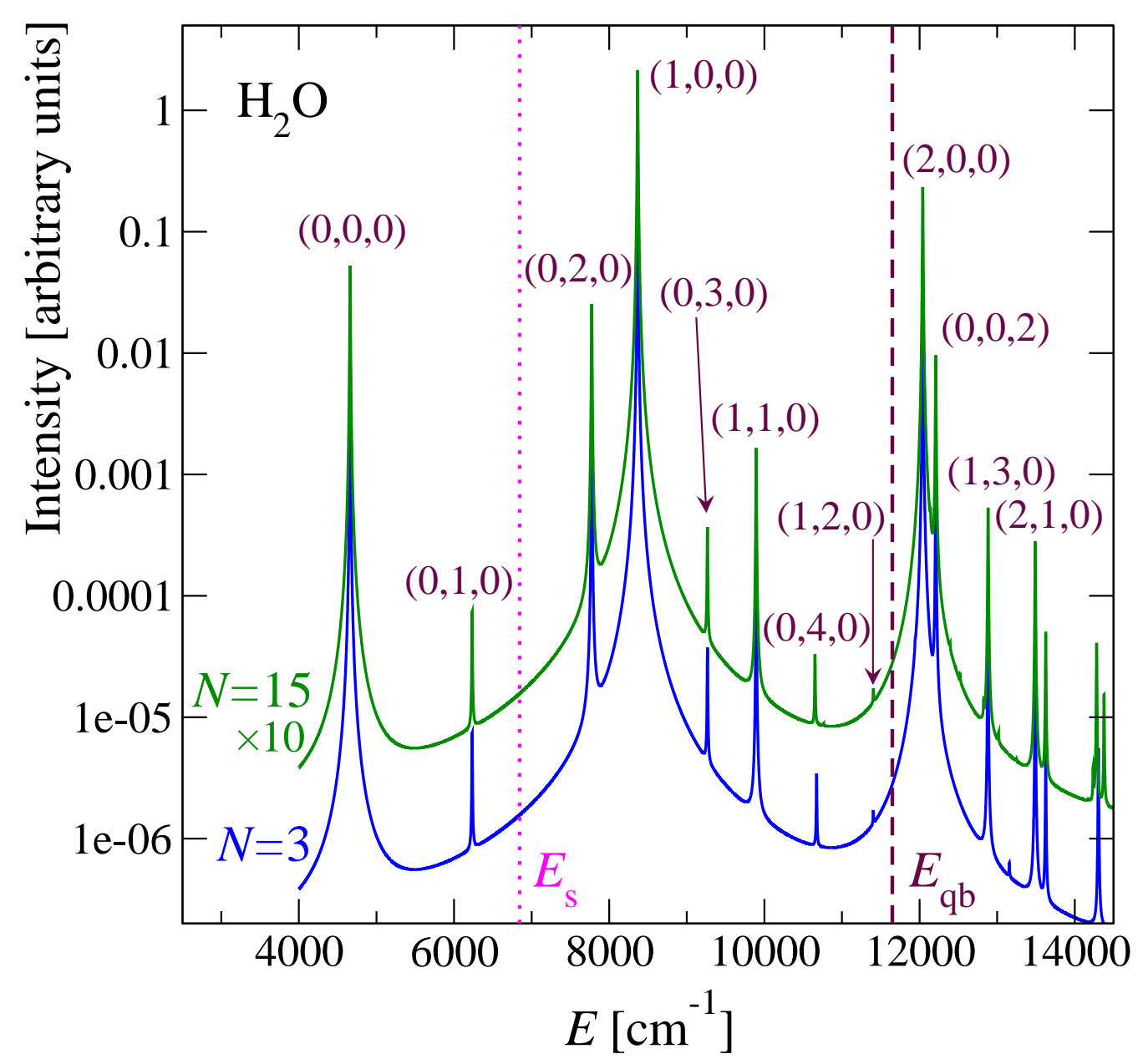

Figure 2 


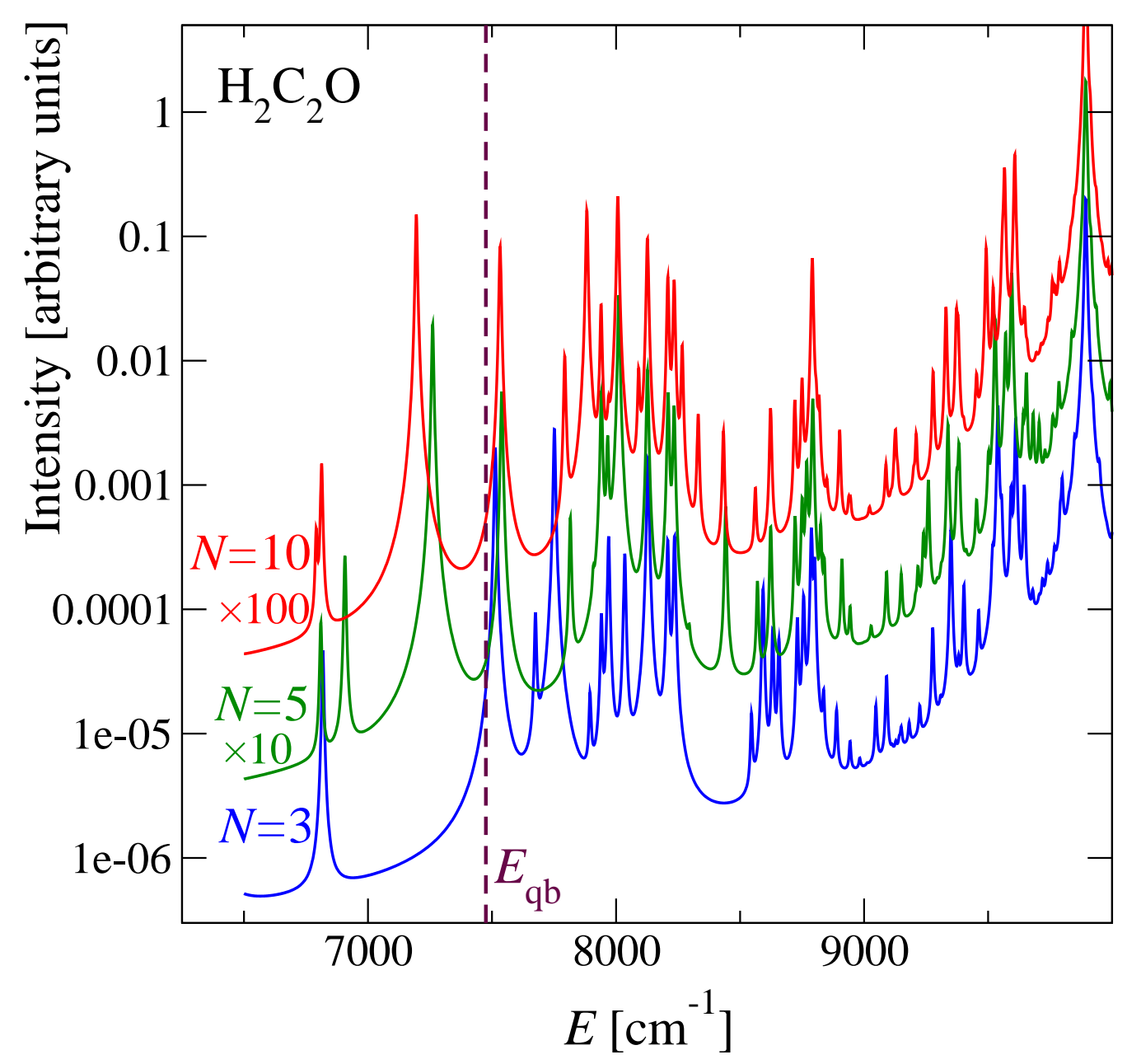

Figure 3 